\title{
FEASST: Free Energy and Advanced Sampling Simulation Toolkit
}

\author{
Harold W. Hatch, Nathan A. Mahynski, and Vincent K. Shen \\ National Institute of Standards and Technology, \\ Gaithersburg, MD 20899 \\ harold.hatch@nist.gov
}

Software DOI: https://doi.org/10.18434/M3S095

Software Version: 1.0

Key words: advanced sampling, flat histogram, free energy, molecular simulation software, Monte Carlo.

Accepted: February 23, 2018

Published: March 1, 2018

https://doi.org/10.6028/jres.123.004

\section{Summary}

The Free Energy and Advanced Sampling Simulation Toolkit (FEASST) is a free, open-source, modular program to conduct molecular and particle-based simulations with Metropolis, Wang-Landau and Transition-Matrix Monte Carlo methods [1-7]. FEASST is implemented in C++ and may be imported as a module within Python 2 or $3 .^{1}$

This document describes the initial public release version 1.0 with the following features:

1. Simulation techniques

- Wang-Landau Monte Carlo

- Transition-matrix Monte Carlo

- Metropolis Monte Carlo

2. Thermodynamic ensembles

- Grand canonical ensemble

- Isothermal isobaric ensemble

- Canonical ensemble

3. Advanced Monte Carlo algorithms

- Parallel configuration swaps

\footnotetext{
${ }^{1}$ Certain commercial firms and trade names are identified in this document in order to specify the installation and usage procedures adequately. Such identification is not intended to imply recommendation or endorsement by the National Institute of Standards and Technology, nor is it intended to imply that related products are necessarily the best available for the purpose.
} 
- Floppy box

4. Intermolecular interactions

- Charged interactions with the Ewald summation

- Lennard-Jones with different exponential parameters, long range corrections, Yukawa, force shifted and/or Gaussians

- Hard spheres, soft spheres and square wells

5. Modern software

- Interface with $\mathrm{C}++$ or as a Python module

- Open Multi-Processing (OpenMP) parallelization

- Check points to save and restart simulations

- Robust unit testing

Many more features are planned for release in future versions of FEASST including expanded ensembles in temperature and alchemical transformations, configurational bias, geometric cluster algorithm, aggregation volume bias, confinement, patchy particle potentials and Mayer-sampling Monte Carlo.

\section{Software Specifications}

\begin{tabular}{|l|l|}
\hline NIST Operating Unit & $\begin{array}{l}\text { Material Measurement Laboratory, Chemical Sciences Division, Chemical Informatics } \\
\text { Group }\end{array}$ \\
\hline Category & Classical molecular and particle-based simulation \\
\hline Targeted Users & Researchers, educators, and students \\
\hline Operating Systems & Cross platform CMake 2.8 build. Automated build testing on Linux and macOS \\
\hline Programming Language & C++11 with optional Python 2.7 or 3.6 \\
\hline Inputs/Outputs & $\begin{array}{l}\text { FEASST interfaces with C++ or Python. It may require many parameters and/or config- } \\
\text { urations typically required by complex molecular simulation code, and FEASST may } \\
\text { output structural and thermodynamic properties (instantaneous and statistic ensemble } \\
\text { averages). }\end{array}$ \\
\hline Documentation & https://doi.org/10.18434/M3S095 \\
\hline Accessibility & N/A, small-scale research tool \\
\hline Disclaimer & https://www.nist.gov/director/licensing \\
\hline
\end{tabular}

\section{Methods for Validation}

The software was validated by reproducing published results, as documented in the website listed above. An extensive suite of unittests for both $\mathrm{C}++$ and Python provides coverage for the majority of lines of code. In addition to unittests, potential memory leaks are detected using Valgrind as part of a nightly automated build. Source code style follows recommendations from Google C++ Style Guide, cpplint, and pylint. 


\section{Journal of Research of National Institute of Standards and Technology}

\section{Acknowledgments}

The authors would like to acknowledge the following people in alphabetical order of last name for discussion and/or testing: Dr. Debra Audus, Dr. Marco A. Blanco, Cesear O. Calero-Rubio, Dr. Joseph Curtis, Dr. Tamoghna Das, Dr. Steven Howell, Sally Jiao, Dr. William P. Krekelberg, Gordon W. McCann, Prof. Jeetain Mittal, Prof. Jeremy Palmer, Prof. Christopher J. Roberts, Prof. Sapna Sarupria, Dr. David A. Sheen, Dr. Daniel W. Siderius, and Dr. Hasan Zerze.

\section{References}

[1] Shen VK, Siderius DW (2014) Elucidating the effects of adsorbent flexibility on fluid adsorption using simple models and flat-histogram sampling methods. Journal of Chemical Physics 140(24):244106. https://doi.org/10.1063/1.4884124.

[2] Hatch HW, Mittal J, Shen VK (2015) Computational study of trimer self-assembly and fluid phase behavior. Journal of Chemical Physics 142(16):164901. https://doi.org/10.1063/1.4918557.

[3] Hatch HW, Yang SY, Mittal J, Shen VK (2016) Self-assembly of trimer colloids: effect of shape and interaction range. Soft Matter 12(18):4170-4179. https://doi.org/10.1039/C6SM00473C.

[4] Hatch HW, Krekelberg WP, Hudson SD, Shen VK (2016) Depletion-driven crystallization of cubic colloids sedimented on a surface. Journal of Chemical Physics 144(19):194902. https://doi.org/10.1063/1.4949758.

[5] Mahynski NA, Zerze H, Hatch HW, Shen VK, Mittal J (2017) Assembly of multi-flavored two-dimensional colloidal crystals. Soft Matter 13(32):5397-5408. https://doi.org/10.1039/C7SM01005B.

[6] Hatch HW, Jiao S, Mahynski NA, Blanco MA, Shen VK (2017) Communication: Predicting virial coefficients and alchemical transformations by extrapolating Mayer-sampling Monte Carlo simulations. Journal of Chemical Physics 147(23):231102. https://doi.org/10.1063/1.5016165.

[7] Shen VK, Siderius DW, Krekelberg WP, Hatch HW (2017) NIST Standard Reference Simulation Website. NIST Standard Reference Database Number 173 (National Institute of Standards and Technology). https://doi.org/10.18434/T4M88Q.

About the authors: H.W.H., N.A.M. and V.K.S. are chemical engineers at NIST. The National Institute of Standards and Technology is an agency of the U.S. Department of Commerce. 